\title{
Phase-sensitive optical processing in silicon waveguides
}

Petermann, Klaus; Gajda, A.; Dziallas, Claudia; Jazayerifar, M.; Zimmermann, L.; Tillack, Bernd; Da Ros, Francesco; Vukovic, Dragana; Dalgaard, Kjeld; Galili, Michael

Total number of authors:

11

Published in:

Proceedings of the Optical Fiber Communication Conference and Exhibition 2015

Link to article, DOI:

10.1364/OFC.2015.Tu2F.4

Publication date:

2015

Document Version

Peer reviewed version

Link back to DTU Orbit

Citation $(A P A)$ :

Petermann, K., Gajda, A., Dziallas, C., Jazayerifar, M., Zimmermann, L., Tillack, B., Da Ros, F., Vukovic, D., Dalgaard, K., Galili, M., \& Peucheret, C. (2015). Phase-sensitive optical processing in silicon waveguides. In Proceedings of the Optical Fiber Communication Conference and Exhibition 2015 [Tu2F.4] IEEE. https://doi.org/10.1364/OFC.2015.Tu2F.4

\section{General rights}

Copyright and moral rights for the publications made accessible in the public portal are retained by the authors and/or other copyright owners and it is a condition of accessing publications that users recognise and abide by the legal requirements associated with these rights.

- Users may download and print one copy of any publication from the public portal for the purpose of private study or research.

- You may not further distribute the material or use it for any profit-making activity or commercial gain

- You may freely distribute the URL identifying the publication in the public portal 


\title{
Phase-sensitive optical processing in silicon waveguides
}

\author{
K. Petermann ${ }^{1}$, A. Gajda ${ }^{1}$, G. Dziallas ${ }^{1}$, M. Jazayerifar ${ }^{1}$, L. Zimmermann ${ }^{2}$, B. Tillack ${ }^{2}$ \\ F. Da Ros $^{3}$, D. Vukovic ${ }^{3}$, K. Dalgaard ${ }^{3}$, M. Galili ${ }^{3}$, C. Peucheret ${ }^{4}$ \\ (1) Technische Universität Berlin, HFT4, Einsteinufer 25, 10587 Berlin, Germany \\ (2) IHP, Im Technologiepark 25, 15236 Frankfurt (Oder), Germany \\ (3) Technical University of Denmark, Department of Photonics Engineering, DK-2800 Kgs. Lyngby, Denmark \\ (4) University of Rennes 1, FOTON Laboratory, CNRS UMR 6082, ENSSAT, F-22305 Lannion, France
}

\begin{abstract}
Parametric optical signal processing is reviewed for silicon nano-rib-waveguides with a reverse-biased pin-junction. Phase-sensitive parametric amplification with a phase-sensitive extinction of more than $20 \mathrm{~dB}$ has been utilized for the regeneration of DPSK signals. OCIS codes: (130.3120) Integrated optics devices; (190.4360) Nonlinear optics devices; (190.4975) Parametric processes; (230.7405) Wavelength conversion devices
\end{abstract}

\section{Introduction}

The use of parametric processes using the $3^{\text {rd }}$ order optical nonlinearity in optical materials for applications such as low noise amplification, wavelength conversion, signal regeneration and other kinds of optical signal processing has raised considerable interest over the last years [1-3]. In particular, parametric processes also allow for phasesensitive optical amplification, which may be utilized for signal regeneration [4], even for higher order modulation formats [5]. Most experiments have so far been conducted using highly nonlinear silica fibers (HNLFs), which require a length of typically several hundred meters due to the relatively low optical nonlinearity in silica.

In order to achieve compact modules, $3^{\text {rd }}$ order nonlinearities may also be utilized in photonic integrated circuits (PICs). For this purpose, PICs have been either based on glasses or semiconductor materials. With respect to PICs based on glass materials, nonlinear parametric processing has been demonstrated, e.g. for silicon nitride, Hydex [6] or chalcogenide glasses [7]. Even though the nonlinearities in these waveguides are about two orders of magnitude higher than in silica-HNLFs, they usually still require pump powers in excess of several Watts because of the relatively short interaction lengths in PICs. Nevertheless, it has been possible to achieve a phase-sensitive extinction ratio of around $10 \mathrm{~dB}$ in a dual-pump degenerate-idler configuration with planar chalcogenide glass waveguides [8] in pulsed operation.

Much higher nonlinearities are feasible in PICs based on semiconductor materials, where silicon is of particular interest [9]. It combines the advantage of a relatively high $3^{\text {rd }}$ order nonlinearity with the realization of nanowires with tiny effective cross sections down to about $0.1{\mu \mathrm{m}^{2}}^{2}$ exhibiting nonlinear phase shifts of around 200...300 rad/(W·m), which is about 4 orders of magnitude higher than in silica HNLFs. Even higher effective nonlinearities are possible for photonic crystal Si-nano-waveguides thanks to slow light enhancement, where, for a phase-sensitive amplifier in pulsed operation, a phase-sensitive extinction ratio of $11 \mathrm{~dB}$ has been achieved [10].

However, silicon suffers from two-photon-absorption (TPA) and the associated generation of carriers leading to free-carrier absorption (FCA). TPA can be reduced using amorphous instead of crystalline silicon [11] or silicon-slot waveguides employing polymers on a silicon-organic platform [12]. However, both concepts suffer from relatively high linear losses and a limited compatibility with CMOS or BiCMOS micro-electronics. TPA can also be omitted by operating the devices at longer infrared wavelengths $[13,14]$. The influence of FCA can be reduced for operation with pulsed pump sources, which is, however, not adequate for telecom applications. Alternatively, FCA in crystalline silicon nano-waveguides can be considerably reduced by reducing the effective carrier lifetime down to about 10-20 ps via a reverse biased pin-junction [15-17].

In this paper, we review our recent achievements on the design and fabrication of silicon nanowires using reverse-biased pin junctions and their applications for phase-sensitive all-optical signal processing at telecom wavelengths using cw-pump sources.

\section{Silicon nano-waveguide design}

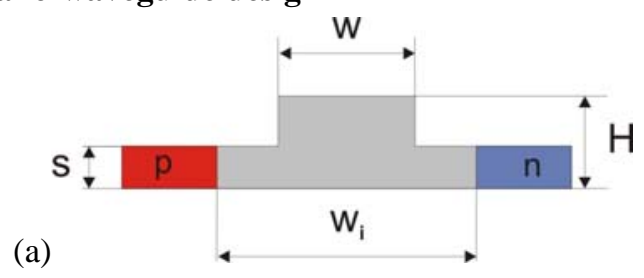

(b)

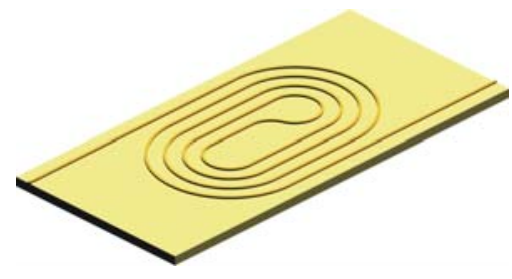

Fig. 1. Structure of the used nano-rib waveguide (a) and its realization with a spiral (b) for a small footprint. 
In order to combine Si-nano-waveguides with a pin-junction, nano rib-waveguides have been fabricated according to Fig. 1 on $2 \mu \mathrm{m}$ thick buried oxide with $\mathrm{W}=500 \mathrm{~nm}, \mathrm{H}=210 \mathrm{~nm}, \mathrm{~s}=50 \mathrm{~nm}$ and $\mathrm{w}_{\mathrm{i}}=1200 \mathrm{~nm}$ within the BiCMOS foundry of IHP using a $248 \mathrm{~nm}$ lithography with a linear loss of about $1 \mathrm{~dB} / \mathrm{cm}$ [18]. The waveguide is realized in form of a spiral yielding a small footprint of only $2.5 \times 0.6 \mathrm{~mm}^{2}$ for a waveguide with a length of $4 \mathrm{~cm}$.

\section{Wavelength conversion with four-wave mixing}

Si rib waveguides with a reverse biased pin-junction allow for very efficient wavelength conversion via fourwave mixing $[15,18,19]$. As shown in Fig. 2, a record cw conversion efficiency (defined as the ratio between idler and signal power at the waveguide output) of $-1 \mathrm{~dB}$ has been achieved (best value of $-0.7 \mathrm{~dB}$ ) [18].
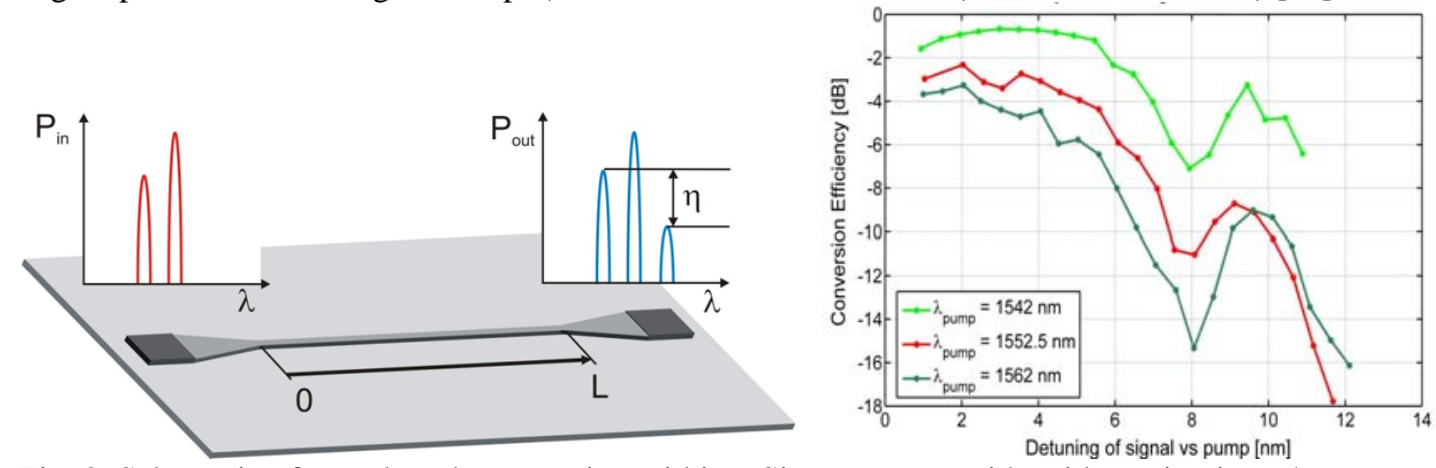

Fig. 2. Schematic of wavelength conversion within a Si-nano-waveguide with grating input/output-couplers and measurement results (length of the waveguide $\mathrm{L}=4 \mathrm{~cm}$, loss per grating coupler $\sim 4.5 \mathrm{~dB}$, cw-pump power before the grating $26 \mathrm{dBm}$, reverse bias voltage $20 \mathrm{~V}$ ) [18].

This high conversion efficiency was achieved for just $26 \mathrm{dBm}$ of external cw-pump power, corresponding to a pump power inside the waveguide of only about $150 \mathrm{~mW}$. The conversion bandwidth is limited by the relatively high amount of chromatic dispersion, which we estimate to about $-2.5 \mathrm{ps} /(\mathrm{m} \cdot \mathrm{nm})$ for our specific waveguide [20,21].

These results underline the high efficiency of four-wave mixing processes in Si nano-waveguides, which have also been exploited for error-free wavelength conversion of $40 \mathrm{Gbit} / \mathrm{s}$ on-off keying (OOK) modulated signals [21].

\section{Phase-sensitive optical signal processing}

Based on the high efficiency of four-wave mixing processes in silicon waveguides, it is also very attractive to study phase-sensitive parametric processing in these waveguides with the purpose of signal regeneration of phase modulated signals. We did investigate both phase-sensitive parametric gain as well as the regeneration of $10 \mathrm{Gbit} / \mathrm{s}$ differential phase-shift keying (DPSK) signals [22, 23] with a set-up as shown in Fig.3.

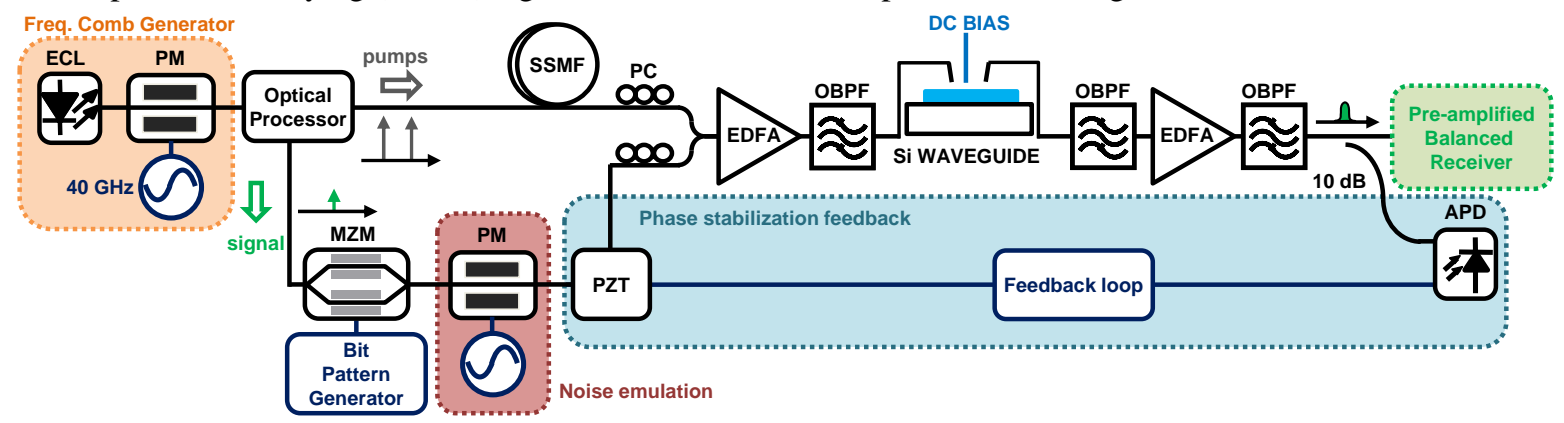

Fig. 3 Experimental set-up for the dynamic phase regeneration of a $10 \mathrm{Gbit} / \mathrm{s}$ DPSK-signal [23].

A frequency comb is being synthesized with a phase-modulator (PM) with a $40 \mathrm{GHz}$ sinusoidal drive signal. The optical processor selects three neighboring comb lines. The two outer comb lines act as phase synchronized pumps and the central line serves as the signal. Fig. 4a shows the measured signal power at the output of the waveguide as a function of the signal phase. For a total cw-pump power $\mathrm{P}_{\mathrm{T}}$ of $24 \mathrm{dBm}$ before the grating (corresponding to $\sim 50 \mathrm{~mW}$ per pump inside the waveguide) and a reverse bias of $20 \mathrm{~V}$, one obtains a phase-sensitive extinction ratio of $11 \mathrm{~dB}$, which can be improved by increasing the pump power to $\mathrm{P}_{\mathrm{T}}=28 \mathrm{dBm}$ ( $\sim 110 \mathrm{~mW}$ per pump inside the waveguide) and by increasing the reverse bias voltage to $25 \mathrm{~V}$ (for reducing the effective carrier lifetime) to a record extinction ratio up to $20 \mathrm{~dB}$ [23]. This phase-sensitive gain has been used for the regeneration of $10 \mathrm{Gbit} / \mathrm{s}$ DPSK signals. As shown in Fig. 3, the DPSK signal is first generated with a Mach-Zehnder modulator (MZM) and is subsequently 
distorted by a harmonically driven ( $\mathrm{f}=5 \mathrm{GHz}$ ) phase modulator. Excellent regeneration results have been obtained for a cw-pump power of $24 \mathrm{dBm}$ and a reverse bias voltage of $25 \mathrm{~V}$, as shown in Fig. 4b.
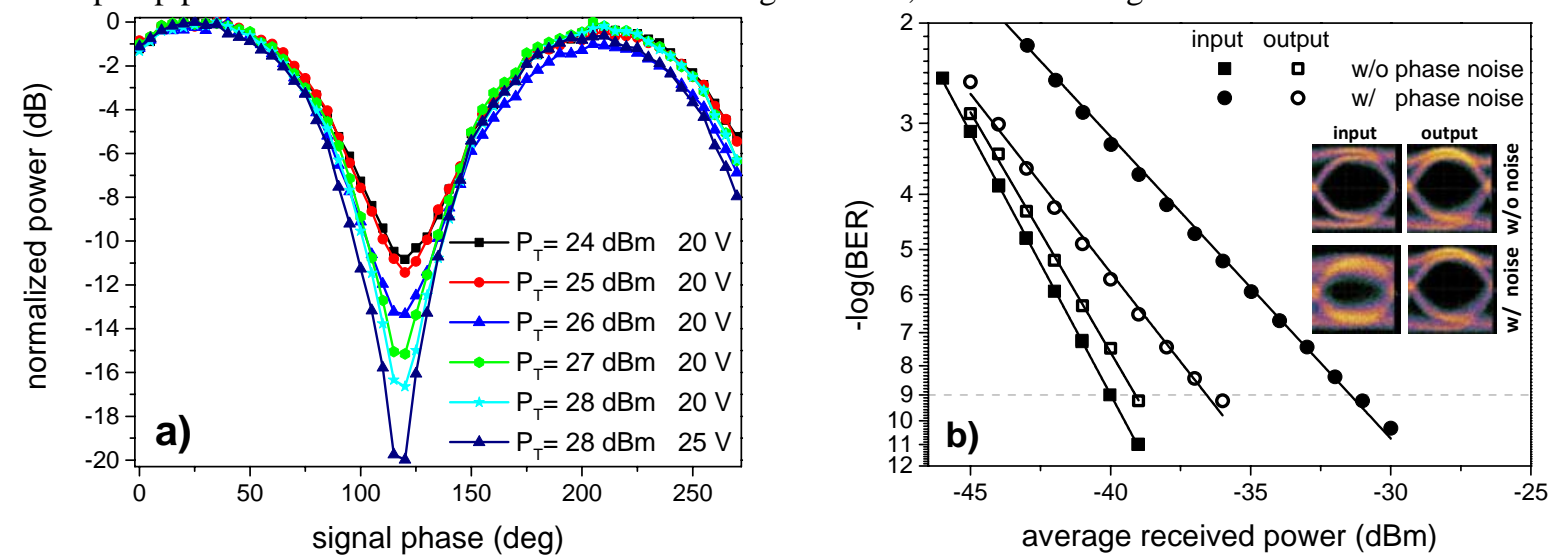

Fig. 4. Phase-sensitive output power (a) and BER curves for a regenerated 10 Gbit/s DPSK signal (b) [23]

\section{Conclusion}

Nano-rib-waveguides with a reverse biased pin-junction are very suitable for advanced parametric signal processing with relatively low cw-pump powers in the order of $100 \mathrm{~mW}$ despite the existence of TPA in silicon at telecommunications wavelengths. Further improvement is expected by suitably tailoring the chromatic dispersion and further reducing the linear losses in the nano-rib-waveguides.

\section{Acknowledgement}

This work was partly funded by the German Research Foundation (DFG) in the framework of the Sonderforschungsbereich SFB787 and by the Danish Research Council for Technology and Production Sciences (project 09-066562). Financial support from Villum Fonden via the NATEC Centre is acknowledged.

\section{References}

[1] J. Hansryd et al., "Fiber-based optical parametric amplifiers and their applications,” IEEE J. Sel. Top. Quantum Electron. 8, 506-520 (2002).

[2] Z. Tong, S.Radic, "Low-noise optical amplification and signal processing in parametric devices,” Adv. Opt. Photon. 5, 318-384 (2013).

[3] M.E. Marhic, Fiber optical parametric amplifiers, oscillators and related devices (Cambridge University Press, 2007)

[4] R Slavík et al., "All-optical phase and amplitude regenerator for next-generation telecommunications systems,” Nature Photon. 4, 690-695 (2010)

[5] T. Richter et al., "QAM Phase regeneration in a phase-sensitive fiber-amplifier," in Proc. ECOC 2013 (IEE, London, 2013), We.3.A.2

[6] D.J. Moss et al., "New CMOS-compatible platforms based on silicon nitride and Hydex for nonlinear optics," Nature Photon. 7, 597-607

(2013).

[7] B.J. Eggleton et al., “Chalcogenide photonics,” Nature Photon. 5, 141-148 (2010).

[8] R. Neo et al., "Phase-sensitive amplification of light in a chi(3) photonic chip using a dispersion engineered chalcogenide ridge waveguide," Opt. Express 21, 7926-7933 (2013).

[9] J. Leuthold et al., “Nonlinear silicon photonics,” Nature Photon. 4, 535-544 (2010).

[10] Y. Zhang et al., "Phase sensitive amplification in silicon photonic crystal waveguides," Opt. Letters 39, 363-366 (2014).

[11] C. Grillet et al., “Amorphous silicon nanowires combining high nonlinearity, FOM and optical stability,” Opt. Express 20, 22609-22615

(2012).

[12] C. Koos et al., "All-optical high-speed signal processing with silicon-organic hybrid slot waveguides,” Nature Photon. 3, 216-219 (2009).

[13] X. Liu et al., "Mid-infrared optical parametric amplifier using silicon nanophotonic waveguides," Nature Photon. 4, 557-560 (2010).

[14] B. Kuyken et al., “50 dB parametric on-chip gain in silicon photonic wires,” Opt. Letters 36, 4401-4403 (2011).

[15] W. Mathlouthi et al., "Characterization of efficient wavelength conversion by four-wave mixing in sub-micron silicon waveguides," Opt. Express 16, 16735-16745 (2008).

[16] A.C. Turner-Foster et al., "Ultrashort free-carrier lifetime in low-loss silicon nanowaveguides,” Opt. Express 18, 3582-3591 (2010).

[17] A. Gajda et al., "Design rules for p-i-n diode carriers sweeping in nano-rib waveguides on SOI,” Opt. Express 19, 9915-9922 (2011).

[18] A. Gajda et al., "Highly efficient CW parametric conversion at $1550 \mathrm{~nm}$ in SOI waveguides by reverse biased p-i-n junction," Opt. Express 20, 13100-13107 (2012).

[19] J. R. Ong et al.,” Efficient CW four-wave mixing in SOI micro-rings with active carrier removal”, IEEE Photon. Technol. Lett. 25, 16991701 (2013)

[20] G. Dziallas et al., "A numerical investigation of continuous wave parametric gain in silicon nano-waveguides at wavelengths around 1550 nm,” in Proceedings NUSOD 2014, paper TuB3, Palma de Mallorca, Spain, 2014.

[21] A. Gajda et al., "High efficiency wavelength conversion of 40 Gbps signals at $1550 \mathrm{~nm}$ in SOI nano-rib waveguides using p-i-n diodes," IEEE Int. Conf. Group IV Photonics, paper FC3, Seoul, Korea, 2013.

[22] F. Da Ros et al., "Continuous wave phase-sensitive four-wave mixing in silicon waveguides with reverse-biased p-i-n junctions," in Proc. ECOC 2013 (IEE, London, 2013), P.2.11

[23] F. Da Ros et al., "Phase regeneration of DPSK signals in a silicon waveguide with reverse-biased p-i-n junction,” Opt. Express 22, 50295036 (2014) 